\title{
Serotipificación de aislamientos clínicos y del medio ambiente de Cryptococcus neoformans en Colombia
}

\author{
Ordóñez N. ${ }^{1}$, Castañeda E. ${ }^{2}$
}

\begin{abstract}
Resumen
Se determinó la variedad y el serotipo de 192 aislamientos de Cryptococcus neoformans recuperados de 1972 a 1992; 163 de origen clínico y 29 del medio ambiente. En los aislamientos clínicos se determinaron las dos variedades de $C$. neoformans: la variedad neoformans en el $92,6 \%$ de los pacientes y la variedad gattii en el $7,4 \%$. En el medio ambiente se encontró la variedad neoformans en el $100 \%$ de los aislamientos. El serotipo encontrado con más frecuencia fue el serotipo $A$, el cual se observó en el $92 \%$ de los aislamientos clínicos y en el $100 \%$ del medio ambiente, seguido por el serotipo B $(6,8 \%)$; también, se determinaron aislamientos de los serotipos $C(0,6 \%)$ y D $(0,6 \%)$. C. neoformans var. neoformans se recuperó en todos los años de los que se tuvieron aislamientos y $C$. neoformans var. gattii solamente se observó en aislamientos clínicos a partir de 1986. C. neoformans var. neoformans fue aislado durante los 12 meses del año y $C$. neoformans var. gattii se encontró distribuido en los meses de febrero, abril, junio, agosto, septiembre y diciembre. C. neoformans var. neoformans se observó en $109(66,9 \%)$ cepas de pacientes del sexo masculinoy en $36(22,1 \%)$ del sexo femenino; C. neoformans var. gattii se observó en $5(3 \%)$ cepas de hombres y en $6(3,7 \%)$ mujeres.
\end{abstract}

\section{Summary}

The variety and serotype of 192 isolates of Cryptococcus neoformans isolated between 1972 and $1992-163$ of clinical origin and 29 of environmental origin - was determined. In the clinical isolates two varieties of $C$. neoformans were identified: $C$. neoformans var. neoformans appeared in $92.6 \%$ of the patients and $C$.neoformans var. gattii in the other 7.4\%. C.neoformans var. neoformans was found in $100 \%$ of the environmental isolates.

Serotype A was most frequently encountered, being observed in $92 \%$ of the clinical isolates and in $100 \%$ of the environmental isolates, followed by serotype B $(6.8 \%)$. Serotype C $(0.6 \%)$ and D $(0.6 \%)$ isolates were also identified.

C. neoformans var. neoformans was seen in every year during which isolates were recorded but $C$. neoformans var. gattii was only observed in clinical isolates from 1986 onwards. C. neoformans var. neoformans was isolated throughout the whole year and C. neoformans var. gattii was encountered only in the months of February, April, June, August, September and December. C. neoformans var. neoformans was observed in 109 strains $(66.9 \%)$ from male patients and in 36 strains $(22.1 \%)$ from female patients. C. neoformans var. gattii was observed in 5 strains (3\%) from men and 6 (3.\&\%) from women.

M.Sc., Grupo de Microbiología, Instituto Nacional de Salud.

2 Ph.D., Grupo de Mlcrobiología, Instituto Nacional de Salud. 
La criptococosis es una micosis oportunista de distribución mundial $(1,2)$. Antes de la aparición del síndrome de inmunodeficiencia adquirida (SIDA), el $50 \%$ de los casos se presentaban en pacientes con alguna condición de base como enfermedades linforreticulares, enfermedades autoinmunes y tratamientos prolongados con corticosteroides $(1,2)$; a partir de 1980 y como consecuencia del SIDA, ha ocurrido un incremento mundial en la incidencia de la enfermedad (3). El agente etiológico de la criptococosis es la levadura de la clase Basidiomycetes (4) denominada Cryptococcus neoformans. Esta levadura por primera vez se aisló del medio ambiente a partir de suelo contaminado con excretas de palomas. Esta experiencia la han reproducido numerosos autores (5-8) y se acepta que la principal forma como se adquiere la criptococosis, tanto humana como animal, es por la inhalación de material de suelo contaminado con excretas de palomas y de otras aves (5-8).

C. neoformans ha sido clasificado en dos variedades y cuatro serotipos: Cryptococcus neoformans var. neoformans (serotipos A y D) y C. neoformans var. gattii (serotipos B y C) (9). Las dos variedades presentan diferencias desde el punto de vista de composición química de la cápsula, metabolismo, teleomorfo y distribución geográfica de los aislamientos clínicos (9-13); C. neoformans var. neoformans, la más frecuentemente recuperada, presenta una distribución mundial y $C$. neoformans var. gattii se ha aislado en regiones tropicales y subtropicales (10-13). Es de anotar que los aislamientos de pacientes con SIDA, son en su gran mayoría $C$. neoformans var. neoformans, aún cuando procedan de regiones en donde $C$. neoformans variedad gattii presenta una alta prevalencia $(11,14,15)$.

Los estudios de serotipificación de aislamientos de excretas de palomas realizados hasta 1990 , demostraron que todos eran $C$. neoformans var. neoformans serotipo $A(10,12,16,17)$. Recientemente, Ellis logró el aislamiento de $C$. neoformans var. gattii de detritus asociados con Eucalyptus camaldulensis y E. tereticornis y ha propuesto al Eucalyptus como el nicho ecológico para esta variedad $(18,19)$.
Este trabajo se realizó para determinar las variedades y serotipos de las cepas de Cryptococcus neoformans de origen humano y del medio ambiente, aisladas en el país en los años de 1972 a 1992 y para destacar la distribución de las variedades y serotipos con relación a la edad, sexo y procedencia de los pacientes, al año y mes de aislamiento del microorganismo y al resultado de la prueba serológica para determinar anticuerpos contra el virus de la inmunodeficiencia humana $(\mathrm{VIH})$. Los aspectos clínicos y de diagnóstico por el laboratorio, de los aislamientos realizados entre 1972 y 1986, habían sido informados previamente $(20,21)$.

\section{Materiales y métodos}

\section{- Aislamientos clínicos}

Se estudiaron 163 aislamientos clínicos de $C$. neoformans, recuperados entre 1972 y 1992; 114 aislados en el Instituto Nacional de Salud y 49 en otros centros de salud del país de donde fueron remitidos para este estudio. De la Corporación para Investigaciones Biológicas (CIB) de Medellín se recibieron 31 aislamientos, del Hospital Erasmo Meoz de Cúcuta 6 , del Instituto de los Seguros Sociales de Cali 10 y de la Universidad del Valle 2. De diez pacientes se estudiaron 2 aislamientos.

De cada cepa se anotó: fecha del aislamiento, origen, procedencia, edad y sexo del paciente y el resultado de la prueba serológica para el virus de inmunodeficiencia humana $(\mathrm{VIH})$ cuando ésta se pudo realizar.

\section{- Cepas control}

Como controles se emplearon las cepas 68A y $52 \mathrm{D}$ de $C$. neoformans var. neoformans y $112 \mathrm{~B}$ y $18 \mathrm{C}$ de $C$. neoformans var. gattii, gentilmente suministradas por la doctora Jean Kwon-Chung del Instituto Nacional de Salud de los Estados Unidos.

- Muestras del medio ambiente: recolección y procesamiento.

Se recolectaron 146 muestras de tierra, 52 de excretas de palomas recogidas en palomares y 6 de excretas de otras aves, canarios y codornices. La elección de los sitios de recolección por departamento se hizo con base en 
la incidencia de criptococosis humana y a la facilidad de su recolección.

Las muestras en cantidades de $10 \mathrm{~g}$ fueron recolectadas con bajalenguas y colocadas en bolsas plásticas, las cuales se almacenaron en refrigeración por un tiempo no mayor de un mes. Para su procesamiento se colocaron 5 $\mathrm{g}$ de cada muestra en tubos de centrífuga de $50 \mathrm{ml}$, se adicionaron $20 \mathrm{ml}$ de solución tampon, fosfato salino (PBS) estéril y se agitaron en vórtex durante 10 minutos; después de 5 minutos de sedimentación, se tomaron $5 \mathrm{ml}$ de sobrenadante de todas las alturas del tubo y a éstos se les adicionaron $100 \mu \mathrm{l}$ de una solución de penicilina y estreptomicina a una concentración de $20 \mathrm{U}$ cada uno (22). $100 \mu \mathrm{l}$ de esta suspensión se sembraron en ácido cafeico y/o semillas de Guizotia (23). Cada muestra se sembró por duplicado y se incubó a $27^{\circ} \mathrm{C}$ por 3 semanas. Las cajas se observaron diariamente y las colonias de color café, sugestivas del hongo debido a la actividad de la enzima fenol-oxidasa (24), se resembraron en agar glucosado de Sabouraud.

- Pruebas de identificación y/o confirmación

Se basaron en la presencia de cápsula, crecimiento a $37^{\circ} \mathrm{C}$, presencia de las enzimas ureasa (25) y fenoloxidasa (24), ausencia de la enzima nitrato reductasa (26) y patrón de asimilación de carbohidratos en el medio de Wickerham modificado (27).

- Determinación de la variedad

Se realizó en el medio de canavanina glicina azul de bromotimol sódico (CGB), el cual se incubó a $27^{\circ} \mathrm{C}$ durante 5 días, realizando lecturas diarias (9).

- Serotipificación

El serotipo se determinó por aglutinación en lámina (17), empleando antisueros específicos obtenicios en conejos, inoculados intravenosamente con levaduras formalinizadas de cada serotipo, según el esquema de Kwon-Chung (comunicación personal). Los antisueros se purificaron por absorción con los antígenos heterólogos y se titularon con las cepas control (17). El título de los antisueros después de las absorciones fue de 1:16 para el serotipo A, 1:128 para el serotipo $C$ y 1:64 para el serotipo D. El antisuero preparado contra el serotipo B se empleó sin diluir $(1: 1)$.

\section{Resultados}

- Características de los aislamientos clínicos Las 163 cepas se aislaron entre los años de 1972 a $1992 ; 127(77,9 \%)$ fueron aislamientos de los últimos 5 años, 21 (12,9\%) de los años comprendidos entre 1985 y 1987 y 15 $(9,2 \%)$ de 1972 a 1984 . La procedencia de los pacientes se presenta en la tabla 1.

Tabla 1. Distribución de los aislamientos de Cryptococcus neoformans según procedencia de los pacientes

\begin{tabular}{|c|c|c|}
\hline \multirow[t]{2}{*}{ Procedencia } & \multicolumn{2}{|c|}{ Aislamientos } \\
\hline & No. & $\%$ \\
\hline Santafé de Bogotá & 45 & 27,10 \\
\hline Antioquia & 30 & 18,40 \\
\hline Valle & 12 & 7,36 \\
\hline Cundinamarca & 12 & 7,36 \\
\hline Tolima & 7 & 4,29 \\
\hline Norte de Santander & 6 & 3,68 \\
\hline Santander & 3 & 1,84 \\
\hline Nariño & 3 & 1,84 \\
\hline Huila & 2 & 1,23 \\
\hline Casanare & 2 & 1,23 \\
\hline Caldas & 2 & 1,23 \\
\hline Bolivar & 1 & 0,61 \\
\hline Caquetá & 1 & 0,61 \\
\hline Providencia & 1 & 0,61 \\
\hline Arauca & 1 & 0,61 \\
\hline Guaviare & 1 & 0,61 \\
\hline Boyacá & 1 & 0,61 \\
\hline Vichada & 1 & 0,61 \\
\hline Vaupés & 1 & 0,61 \\
\hline Magdalena & 1 & 0,61 \\
\hline Meta & 1 & 0,61 \\
\hline SD & 29 & 17,79 \\
\hline Total & 163 & 100,00 \\
\hline
\end{tabular}

$\mathrm{SD}=\sin$ dato 
- Características de los aislamientos del medio ambiente

Se aislaron 29 cepas de $C$. neoformans; 1 de las $146(0,68 \%)$ muestras de tierra y 28 de las $52(53,8 \%)$ muestras de excretas de palomas; no se obtuvieron aislamientos de las excretas de otras aves. El único aislamiento de tierra fue recuperado de una muestra de Cali, de las muestras de excretas se obtuvieron 11 cepas de Cali, 8 de Cúcuta, 5 de municipios de Cundinamarca y 4 de Santafé de Bogotá.

- Identificación de los aislamientos

En las 192 cepas se observó presencia de cápsula, crecimiento a $37^{\circ} \mathrm{C}$, presencia de la enzima ureasa, ausencia de nitrato reductasa y el patrón para asimilación de azúcares descrito para las especies de C. neoformans, el cual no asimila melibiosa ni lactosa. Solamente en un aislamiento clínico no se pudo demostrar la presencia de la enzima fenoloxidasa.

- Distribución de variedades y serotipos

El cultivo en el medio CGB para la determinación de las variedades permitió demostrar $C$. neoformans var. neoformans en 151/163 $(92,6 \%)$ aislamientos clínicos y en todos los aislamientos del medio ambiente y $C$. neoformans var. gattiien $12 / 163(7,4 \%)$ aislamientos clínicos. El serotipo más abundante fue el $\mathrm{A}$, encontrado en el $92 \%$ de los aislamientos clínicos y en el $100 \%$ del medio ambiente (tabla 2). Los resultados de la determinación de la variedad en el medio CGB concordaron en todos los casos con los de la serotipificación.

Tabla 2. Distribución de los aislamientos de Cryptococcus neoformans según variedades y serotipos.

\begin{tabular}{lllrr}
\hline \multirow{2}{*}{ Origen } & \multicolumn{1}{c}{ Variedad } & Serotipo & No. & $\%$ \\
\hline Clínico & C. neoformans & A & $150 / 163$ & 92,0 \\
& var. neoformans & D & $1 / 163$ & 0,6 \\
\cline { 2 - 5 } & C. neoformans & B & $11 / 163$ & 6,8 \\
& var. gattii & C & $1 / 163$ & 0,6 \\
\hline Medio & C. neoformans & & & \\
ambiente & var. neoformans & A & $29 / 29$ & 100 \\
\hline
\end{tabular}

Distribución de variedades y serotipos según sexo y edad de los 163 pacientes

C. neoformans var. neoformans se observó en $109(66,9 \%)$ pacientes del sexo masculino, en $36(22,1 \%)$ del femenino y en $6(3,7 \%)$ pacientes sin dato; $C$. neoformans var. gattii se observó en $5(3,1 \%)$ pacientes masculinos, en $6(3,7 \%)$ femeninos y en $1(0,6 \%)$ sin dato. En el sexo masculino se observaron los 4 serotipos y en el femenino solamente el $\mathrm{A}$ y el B.

- C. neoformans variedad neoformans

Se observó en todos los grupos de edad, tanto en el sexo masculino como el femenino; $C$. neoformans var. gattii se observó en el grupo de edad de 40 a 79 en el sexo masculino y de 0 a 59 años en el femenino (tabla 3).

- Distribución de las variedades y serotipos según la procedencia de los 163 pacientes

C. neoformans var. neoformans serotipo A mostró una amplia distribución en el país (tabla 4); se observó como variedad y serotipo único en Antioquia, Cundinamarca, Santander, Casanare, Caldas, Bolívar, Caquetá, Providencia, Guaviare, Boyacá, Vichada, Magdalena y Meta. Las dos variedades, serotipos $A$ y B, se encontraron en Valle, Tolima, Nariño, Huila y Norte de Santander; $C$. neoformans var. gattii, serotipos B y $C$ se recuperó de los únicos aislamientos procedentes de Vaupés y Arauca respectivamente.

Tabla 3. Distribución de variedades de Cryptococcus neoformans según sexo y edad de los 163 pacientes

\begin{tabular}{|c|c|c|c|c|c|c|c|}
\hline \multirow{2}{*}{$\begin{array}{l}\text { C. neoforman } \\
\text { variedad }\end{array}$} & \multirow{2}{*}{ is Sexo } & \multicolumn{5}{|c|}{ Grupos de edad } & \multirow{2}{*}{ Total } \\
\hline & & $0-19$ & $20-39$ & $40-59$ & $60-79$ & SD & \\
\hline \multirow[t]{3}{*}{ neoformans } & Masculino & 8 & 38 & 17 & 10 & 36 & 109 \\
\hline & Femenino & 4 & 15 & 4 & 2 & 11 & 36 \\
\hline & $\mathrm{SD}$ & - & - & - & - & 6 & 6 \\
\hline \multirow[t]{3}{*}{ gattii } & Masculino & - & - & 3 & 1 & 1 & 5 \\
\hline & Femenino & 2 & 1 & 2 & - & 1 & 6 \\
\hline & SD & - & - & - & - & 1 & 1 \\
\hline Total & & 14 & 54 & 26 & 13 & 56 & 163 \\
\hline
\end{tabular}

$\mathrm{SD}=\sin$ dato 
Tabla 4. Distribución de las variedades y serotipos de Cryptococcus neoformans según procedencia de los 163 pacientes.

\begin{tabular}{|c|c|c|c|c|}
\hline \multirow[t]{2}{*}{ Procedencia } & \multicolumn{2}{|c|}{$\begin{array}{l}\text { C.neoformans } \\
\text { var. neoformans } \\
\text { serotipo }\end{array}$} & \multicolumn{2}{|c|}{$\begin{array}{l}\text { C. neoformans } \\
\text { var. gatii } \\
\text { serotipo }\end{array}$} \\
\hline & A & D & B & C \\
\hline Antioquia & $30 / 30$ & - & - & - \\
\hline Cundinamarca & $12 / 12$ & - & - & - \\
\hline Santander & $3 / 3$ & - & - & - \\
\hline Casanare & $2 / 2$ & - & - & - \\
\hline Caldas & $2 / 2$ & - & - & - \\
\hline Bolivar & $1 / 1$ & - & - & - \\
\hline Caquetá & $1 / 1$ & - & - & - \\
\hline Providencia & $1 / 1$ & - & - & - \\
\hline Guaviare & $1 / 1$ & - & - & - \\
\hline Boyacá & $1 / 1$ & - & - & - \\
\hline Vichada & $1 / 1$ & - & - & - \\
\hline Magdalena & $1 / 1$ & - & - & - \\
\hline Meta & $1 / 1$ & - & - & - \\
\hline Santafé de Bogotá & $44 / 45$ & $1 / 45$ & - & - \\
\hline Valle & $11 / 12$ & - & $1 / 12$ & - \\
\hline Tolima & $6 / 7$ & - & $1 / 7$ & - \\
\hline Nariño & $2 / 3$ & - & $1 / 3$ & - \\
\hline Huila & $1 / 2$ & - & $1 / 2$ & - \\
\hline Norte Santander & $1 / 6$ & - & $5 / 6$ & - \\
\hline Arauca & - & - & - & $1 / 1$ \\
\hline Vaupés & - & - & $1 / 1$ & - \\
\hline SD & $28 / 29$ & - & $1 / 29$ & - \\
\hline Total & $150 / 163$ & $1 / 163$ & $11 / 163$ & $1 / 163$ \\
\hline
\end{tabular}

$\mathrm{SD}=\sin$ dato

- Distribución de variedades y serotipos de las 163 cepas clínicas según el año y el mes de aislamiento

C. neoformans var. neoformans, serotipo A, se observó en todos los años en los que se tuvieron aislamientos (figura 1) y se encontró como única variedad entre los años de 1972 a 1985 , en 1987 y 1992; el serotipo D se encontró en 1981. C. neoformans var. gattii, serotipo B, solamente se observó a partir de 1986, distribuido en 2/8 aislamientos de 1986 , $1 / 17$ de $1988,3 / 22$ de $1989,3 / 25$ de 1990 y 2/34 de 1991 y el serotipo $C$ en 1/22 de 1989.

Se recuperó $C$. neoformans var. neoformans, serotipo $A$, durante los 12 meses del año (figura 2) y el serotipo $D$ se aisló en diciembre; $C$. neoformans var. gattii, serotipo $B$, se recuperó en $1 / 14$ aislamiento de febrero, 3/ 23 de abril, $1 / 9$ de junio, $3 / 15$ de agosto, $2 / 17$ de septiembre y $1 / 13$ de diciembre; el serotipo C se aisló en agosto.

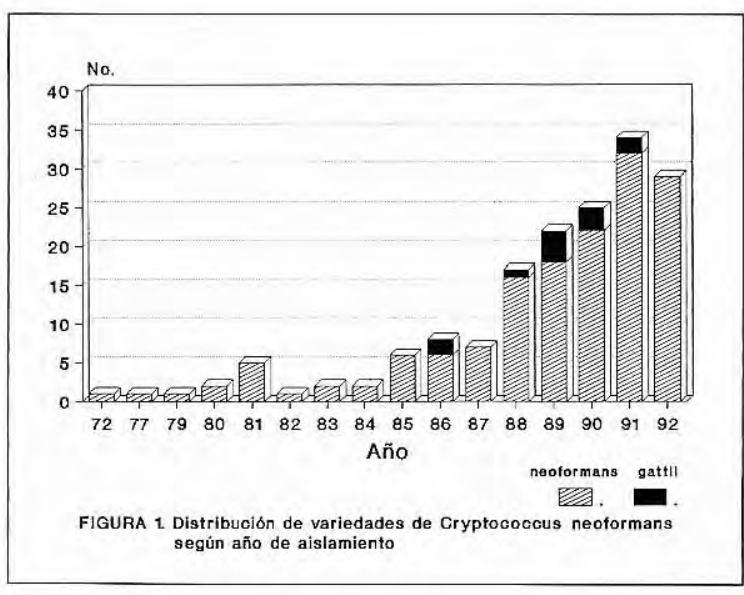

Figura 1. Distribución de variedades de Cryptococcus neoformans, según año de aislamiento.

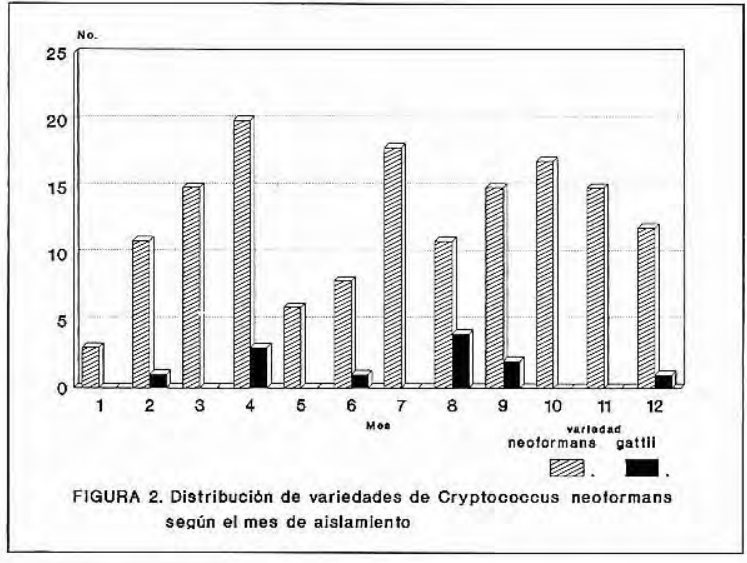

Figura 2. Distribución de variedades de Cryptococcus neoformans, según el mes de aislamiento. 
- Distribución de los aislamientos de Cryptococcus neoformans según el resultado del VIH y sexo de los 163 pacientes

En los 58 pacientes $\mathrm{VIH}$ positivos se aisló $C$. neoformans var. neoformans, serotipo $A ; 19$ de los 26 aislamientos de los pacientes VIH negativos fueron $C$. neoformans var. neoformans serotipo A y 7 C. neoformans var. gattii; 74 de los 79 pacientes con resultado desconocido del $\mathrm{VIH}$ presentaron $C$. neoformans var. neoformans, 73 serotipo A y 1 serotipo Dy 5 C. neoformans var. gattiiserotipo B (tabla 5).

- Cepas aisladas de pacientes en tratamiento

El estudio de 10 aislamientos de C. neoformans var. neoformans serotipo A, de pacientes que ya habían iniciado tratamiento, mostró que éste no alteró los resultados de serotipificación ni la determinación de la variedad en el medio CGB.

Tabla 5. Distribución de los aislamientos de Cryptococcus neoformans según HIV y sexo de los 163 pacientes.

\begin{tabular}{|c|c|c|c|c|c|c|}
\hline \multirow[t]{2}{*}{ HIV } & \multirow[t]{2}{*}{ Sexo } & \multicolumn{4}{|c|}{ Cryptococcus neoformans } & \multirow{2}{*}{ Total } \\
\hline & & var. $n$ & mans & va & $t i i$ & \\
\hline
\end{tabular}

\begin{tabular}{llrllll}
\hline Positivo & M & 52 & 0 & 0 & 0 & \\
& F & 3 & 0 & 0 & 0 & \\
SD & 3 & 0 & 0 & 0 & \\
& Total & 58 & 0 & 0 & 0 & 58 \\
\hline
\end{tabular}

\begin{tabular}{lrrrrrr}
\hline Negativo M & 13 & 0 & 4 & 1 & \\
F & 6 & 0 & 2 & 0 & \\
SD & 0 & 0 & 0 & 0 & \\
Total & 19 & 0 & 6 & 1 & 26 \\
\hline
\end{tabular}

\begin{tabular}{llrrrrr}
\hline SD & $M$ & 43 & 1 & 1 & 0 & \\
& F & 27 & 0 & 3 & 0 & \\
& SD & 3 & 0 & 1 & 0 & \\
& Total & 73 & 1 & 5 & 0 & 79 \\
\hline Total & & 150 & 1 & 11 & 1 & 163 \\
\hline
\end{tabular}

$M=$ masculino

$\mathrm{F}=$ femenino

$\mathrm{SD}=\sin$ dato

\section{Discusión}

No obstante el disponer de aislamientos en un amplio rango de años, la escasa cantidad de cepas de los primeros años estudiados se podría explicar por la menor frecuencia de diagnóstico, sumada a la pérdida de viabilidad de algunas cepas durante su almacenamiento. El número de aislamientos de los últimos años comparados con la revisión de casos colombianos realizada por Greer en 1977, en donde se informaron 53 casos de criptococosis ocurridas entre 1956 y 1977, confirman en general, que actualmente la criptococosis se diagnostica mejor en nuestro medio (1).

Los medios de cultivo empleados permitieron la recuperación relativamente fácil del microorganismo de excretas de palomas, a pesar de lo contaminado de las muestras; este hecho había sido descrito en otros trabajos $(8,22,28,29)$. La positividad del $54 \%$ observada en la recuperación del hongo a partir de muestras de excretas de palomas fue superior a la obtenida por otros autores como Pérez (15,5\%), Littman (36\%), Swinne-Desgain $(45 \%)$ y por Mira $(13,8 \%$ ) lo cual puede explicarse por la elección de las muestras apropiadas y la alta eficiencia de recuperación en los medios empleados $(8,22,23,29)$.

El aislamiento de una sola cepa de $C$. neoformans de las muestras de tierra concuerda con lo descrito por Denton (30) para este tipo de muestra, quien también lo aisló en baja cantidad en este materiai; se desconoce la razón de la presencia del microorganismo en tierra aparentemente libre de excretas de palomas; Denton la relacionó con otro hábitat del microorganismo aún no reconocido (30).

En nuestro trabajo no se logró la recuperación del hongo en ninguna de las muestras de excretas de otras aves; sin embargo, el número tan bajo de muestras procesadas nos impide sacar conclusiones. Staib informó sobre aislamientos de C. neoformans de excretas de 4 de 142 especies de aves, diferentes a palomas (28); igualmente, Swinne-Desgain logró recuperar el hongo de excretas de canarios en el 19\% de 200 muestras de excretas estudiadas (29). 
Las pruebas empleadas en la identificación de las cepas permitieron comprobar que todas eran aislamientos de $C$. neoformans, incluyendo la cepa en la que no se determinó la presencia de la fenoloxidasa. Las cepas melanina negativas tipo silvestre, aunque son raras, han sido descritas esporádicamente en la literatura y esta característica, unida a la presencia de cápsula, ha sido correlacionada con la virulencia del hongo (31). Kwon-Chung en 1986, demostró que las cepas capsuladas y melanina positivas eran letales para el $90-100 \%$ de los ratones infectados, durante un período de 40 días después de la infección; por el contrario, con cepas capsuladas melanina negativas, la letalidad fue del 70 al $90 \%$ después de los 40 días de infección (30).

Los resultados de la determinación de la variedad en el medio CGB, concordaron con los resultados de la serotipificación con los antisueros serotipo-específicos, demostrando una vez más la alta especificidad que presenta el medio de CGB (9).

Los porcentajes del $92,6 \%$ de aislamientos clínicos de $C$. neoformans var. neoformans y el $7,4 \%$ de C. neoformans var. gattii son muy similares a los observados en Argentina (32), Brasil (33) y Norteamérica (10), sin incluir el sur de California, pero diferentes al $80 \%$ descrito en Canadá (10), $70 \%$ en Venezuela (34) y $11 \%$ en México (10).

C. neoformans var. neoformans mostró una amplia distribución en Colombia, encontrándose en 18 de los 20 departamentos de donde se tuvieron aislamientos y en Santafé de Bogotá; $C$. neoformans var. gattii se determinó en baja cantidad y solamente en 6 departamentos, pero llama la atención el número de aislamientos de esta variedad en Norte de Santander; desconocemos los motivos por los cuales ésto sucede y actualmente es objeto de estudio.

En las regiones en las cuales se aisló $C$. neoformans var. gattii y se realizaron estudios del medio ambiente, no fue posible correlacionar los hallazgos debido, tal vez, a que el material estudiado fueron excretas de palomas, las cuales constituyen el hábitat de $C$. neoformans var. neoformans $(12,16,17,35)$. Debido a la alta pre- valencia de $C$. neoformans var. gattii en aislamientos clínicos en Australia, Ellis inició el estudio de la ecología de esta variedad y en 1990 logró recuperar la variedad en los detritus del Eucalyptus camaldulensis. Valdría la pena investigar si éste es el sitio en donde habita el $C$. neoformans var. gattii en nuestro medio (18).

El serotipo $A$, aislado con mayor frecuencia en nuestro estudio, ha sido observado también con mayor frecuencia en Canadá, Estados Unidos, Brasil, Venezuela, Japón, Nueva Guinea y algunos países europeos; destacamos la presencia del serotipo $\mathrm{C}$, el cual había sido descrito solamente en Estados Unidos, Canadá y Reino Unido $(10,12,16,34)$; el paciente del cual se obtuvo el aislamiento era un agricultor de Tame, Arauca, quien informó no haber salido del país. El aislamiento del serotipo $D$ de un paciente de Santafé de Bogotá no pudo ser considerado como autóctono, ya que el paciente relató un viaje a Venezuela, a la región del Zulia, en donde había sido descrito un caso de I mismo serotipo por Villanueva y col. (34).

El serotipo A fue el único determinado en los aislamientos del medio ambiente, lo cual concuerda con lo observado por otros autores $(16,17$, $20,35)$ y está de acuerdo con los hallazgos de los aislamientos clínicos.

Los resultados de las variedades y serotipos según el sexo y la edad de los pacientes, demuestran que la criptococosis ocasionada por $C$. neoformans var. neoformans, serotipo A, se presenta con mayor frecuencia en hombres en edades entre 20 a 40 años, lo cual refleja lo observado por otros autores en la distribución de la criptococosis por sexo y edad antes y después de la aparición del SIDA $(3,11)$; aunque son muy pocos los aislamientos clínicos de C. neoformans var. gattii, no se observó predilección por sexo ni edad.

Al analizar la distribución anual de variedades, observamos que a partir de 1986, C. neoformans var. gattii se aisla en nuestro medio; esta observación, con un número constante de casos, está de acuerdo con la afirmación de Kwon-Chung sobre la permanencia de la variedad (36). No pudimos establecer factores ambientales para 
explicar la criptococosis ocasionada por C. neoformans var. gattii en intervalos de 2 meses; el número tan reducido de aislamientos tampoco nos permitió sacar conclusiones al respecto; $\sin$ embargo, es un dato que se debe tener en cuenta para estudios futuros en búsqueda de la levadura del medio ambiente.

Los aislamientos de pacientes VIH positivos fueron todos $C$. neoformans var. neoformans, serotipo A, lo cual concuerda con lo informado en la literatura $(14,15,37,38,39)$. Solamente se han registrado 4 aislamientos de $C$. neoformans var. gattii en pacientes VIH positivos en Zaire, Canadá, Brasil y California $(14,37,38,39)$. En los aislamientos de los grupos de pacientes $\mathrm{VIH}$ y sin dato de $\mathrm{VIH}$, se observaron las dos variedades de $C$. neoformans como había sido encontrado por Shimizu (40).

Los 10 aislamientos de pacientes que ya habían iniciado tratamiento, permitieron observar que éste no alteró los resultados de la variedad ni el serotipo. Esto demuestra que los componentes estructurales de la cápsula, responsables del serotipo, no se alteran con el tratamiento, por lo menos a un nivel que sea detectado en estas técnicas, lo que permite usarlas en la determinación de la variedad de aislamientos de pacientes en tratamiento.

Con base en este estudio, podemos concluir que en Colombia existen las dos variedades de $C$. neoforman, $C$. neoformans var. neoformans y $C$. neoformans var. gattii, siendo la variedad neoformans la más frecuentemente encontrada en aislamientos clínicos y la única aislada del medio ambiente; se observaron los 4 serotipos en los aislamientos clínicos, siendo el serotipo A el más frecuente, seguido por el serotipo $B$ y es la primera vez que se encuentra el serotipo $C$ en Sur américa.

\section{Agradecimientos}

Deseamos expresar nuestros agradecimientos a la doctora Angela Restrepo y a las bacteriólogas de la Corporación para Investigaciones Biológicas ( $\mathrm{CIB}$ ) de Medellín, a las bacteriólogas María Claudia Rodríguez del Hospital Erasmo Meoz de Cúcuta, Lucero de Salazar de los Seguros Sociales de Cali y a María Inés Alvarez de la Universi- dad del Valle, por habernos enviado los aislamientos de sus pacientes para el presente trabajo.

\section{Bibliografía}

1. Greer D, Polanía LA. Criptococosis en Colombia: resumen de la literatura y presentación de doce casos en el Valle del Cauca. Acta Med Valle 1977; 4:160.

2. Salaki J, Louria D, Chmel H. Fungal and yeast infections of the central nervous system. A clinical review. Medicine $1984 ; 63: 108$.

3. Clarck R, Greer D, Atkinson W, et al. Spectrum of Cryptococcus neoformans infection in 68 patients infected with human immunodeficiency virus. Rev Infect Dis 1990; $12: 768$.

4. Alexopoulos C, Mims C. Introductory mycology. Canadá: Wiley J Sons Eds., 1979:414.

5. Emmons CW. Saprophytic sources of Cryptococcus neoformans associated with the pigeon (Columbia livia). Am J Hyg 1955; 62:227.

6. Corrales C, Ordóñez N, Castañeda E. Determinación de anticuerpos contra Cryptococcus neoformans en un grupo de colombófilos. Biomédica 1987; 7:100.

7. Howard W, Hall N, Schiltzer R. Dynamics of cryptococcal infection by the airborne route. In the black and white yeasts. Proceedings of the Fourth International Conference on Mycoses. Pan American Health Organization Scientific Publication 356, Washington D C., 1978; 185.

8. Mira C, Anzola R, Martínez A, et al. Aislamiento de Cryptococcus neoformans a partir de materiales contaminados con excreta de palomas en Medellín, Colombia. Antioquia Med 1968; 18:33.

9. Kwon-Chung KJ, Polacheck I, Bennett JE. Improved diagnostic medium for separation of Cryptococcus neoformans var. neoformans (serotypes $A$ and $D$ ) and Cryptococcus neoformans var. gattii (serotypes B and C). J Clin Microbiol 1982; 15:535.

10. Kwon-Chung KJ, Bennett JE. Epidemiologic differences between the two varieties of Cryptococcus neoformans. Am J Epidemiol 1984; 120:123.

11. Kwon-Chung kJ, Kozel T, Edman J, et al. Recent advances in biology and immunology of Cryptococcus neoformans. J Med Vet Micol 1992; 30:133.

12. Mishra S, Staib F, Folkens V, et al. Serotypes of Cryptococcus neoformans strains isolated in Germany. J Clin Microbiol 1981; 14:106.

13. Walter J, Coffee E. Distribution and epidemiologic significance of serotypes of Cryptococus neoformans. Am J Epidemiol 1968; 87:167.

14. Bottone E, Salkin I, Hurd N, et al. Serogroup distribution of Cryptococcus neoformans in patients with AIDS. J Infect Dis 1987; 156:242.

15. Rinaldi M, Drutz D, Howell D, et al. Serotypes of Cryptococcus neoformans in patients with AIDS. J Infect Dis $1990 ; 153: 642$. 
16. Hironaga M, Ikeda R, Fukazawa $Y$, et al. Mycological note. Mating types and serotypes of Cryptococcus neoformans isolated in Japan. Sabouraudia 1983;21: 73.

17. Kwon-Chung KJ, Bennett JE. High prevalence of Cryptococcus neoformans var.gattii in tropical and subtropical regions. Zbl Bakt Hyg 1984; A257:213-218.

18. Ellis D, Pfeiffer T. Cryptococcus neoformans var. gattii. J Clin Microbiol 1990; 28:1642.

19. Pfeiffer TJ, Ellis DH. Environmental isolation of Cryptococcus neoformans var. gattii from Eucalyptus tereticornis. J Med Vet Micol 1992; 30:407

20. Ordóñez N, Castillo J, Moreno G. Criptococosis: diagnóstico por el laboratorio. Biomédica, 1987; 7: 37.

21. Ordóñez N, Castañeda E. Serotipificación de cepas colombianas de Cryptococcus neoformans aisladas de pacientes. Acta Méd Colomb 1990; 15:261.

22. Pérez S, Gutiérrez J. Aislamiento de Cryptococcusy $C$. neoformans en heces de aves. Rev San Hig Pública 1983; 57:89.

23. Ruiz A, Neilson J, Bulmer G. A one year study on the viability of Cryptococcusneoformansin nature. Mycopathol $1982 ; 77: 117$

24. McCtnis M. Laboratory handbook of medical mycology. New York: Academic Press, 1980.

25. Seeliger $\boldsymbol{H}$. Use of urease test for the screening and identification of Cryptococci. J Bacteriol 1956; 72:127.

26. Hopkins JM, Land GA. Rapid method for determining nitrate utilization by yeasts. J Clin Microbiol 1977; 5:407.

27. Adams ED, Cooper BH. Evaluation of a modified Wickerham medium for identifying medically important yeasts. Amer J Med Technol 1974; 40:377.

28. Staib F, Schulz-Dieterich J. Cryptococcus neoformans in fecal matter of birds kept in cages control of $C$. neoformans habitats. Zbl Bakt Hyg I Abt Orig 1984; B179:179.

29. Swinne-Desgain D. Cryptococcus neoformans of saprophitic origin. Sabouraudia 1975; 13:303.
30. Denton J, Disalvo A. The prevalence of Cryptococcus neoformans in various natural habitats. Sabouraudia 1968; 6:213.

31. Kwon-Chung KJ, Rhodes JC. Encapsulation and melanin formation as indicators of virulence in Cryptococcus neoformans. Infect Immun 1986; 51:218.

32. Bava AJ, Negroni R. Características epidemiológicas de 105 casos de criptococosis diagnosticados en la República Argentina entre 1981-1990. Rev Ins Med Trop Sao Paulo 1992; 31:335.

33. Rozenbaum R, et al. Cryptococcus neoformans varieties as agents of cryptococcosis in Brazil. Mycopatholoy 1992; 119:133.

34. Villanueva E, Mendoza M, Torres E, etal. Serotipificación de 27 cepas de Cryptococcus neoformans aisladas en Venezuela. Acta Científica Venezolana 1989; 40:151

35. Evans E, Kessel J. The antigenic composition of Cryptococcus neoformans. II. Serenbaum R, Rios A, Wanke B, et al. Cryptococcus neoformans var. gattii in a Brazilian AIDS patient. Mycopathology 1990; 112:33.

36. Kwon-Chung KJ, Varma AK, Howard DH. Ecology and epidemiology of Cryptococcus neoformans: a recent study of isolates in the United States. Proceedings of the $X$ Congress of International Society for Human and Animal Mycology. ISHAM, Barcelona, Spain, 1988.

37. Clancy N, Fleishman J, et al. Isolation of Cryptococcus neoformansvar. gattiifrom a patient with AIDS in Southern California. J Infect Dis 1990; 161:809.

38. Rozenbaum R, Rios A, Wanke B, et al. Cryptococcus neoformans var. gattii in a Brazilian AIDS patient. Mycopathology 1990; 112:33.

39. Staib F. Cryptococcus neoformans and cryptococcosis historical background. Proceedings of the $X$ Congress of International Society for Human and Animal Mycology. ISHAM, Barcelona, Spain, 1988.

40. Shimizu R, Howard D, Clancy M. The variety of Cryptococcus neoformans in patients with AIDS. J Infect Dis 1986; 156:1042. 